7th International Workshop on Astronomy and

Relativistic Astrophysics (IWARA 2016)

International Journal of Modern Physics: Conference Series

Vol. 45 (2017) 1760002 (7 pages)

(C) The Author(s)

DOI: $10.1142 / \mathrm{S} 2010194517600023$

\title{
Pseudo-Complex General Relativity
}

\author{
Peter O. Hess \\ Instituto de Ciencias Nucleares, \\ Universidad Nacional Autónoma de México, \\ Apdo. Postal 70-543, México 04510 D.F., Mexico \\ hess@nucleares.unam.mx
}

Published 15 August 2017

\begin{abstract}
The present status of the pseudo-complex General Relativity is presented. The pcGR includes many known theories with a minimal length. Restricting to its simplest form, an energy-momentum tensor is added at the right hand side of the Einstein equations, representing a dark energy, related to vacuum fluctuations. We use a phenomenological ansatz for the density and discuss observable consequences: Quaisperiodic Oscillations (QPO), effects on accretion disks and gravitational waves.
\end{abstract}

Keywords: Pseudo-Complex General Relativity, Accretions Disks, Gravitational Waves.

PACS numbers: 02.40.ky, 98.80.-k

\section{Introduction}

The General Relativity (GR) is one of the best tested theories. ${ }^{1}$ However, the range of very strong fields are barely touched. Only recently the first observations of gravitational waves was reported ${ }^{2-5}$ and one hopes to find further results in near future. ${ }^{6}$ I.e., we are living in very excited times which is also reflected in the topics covered in this meeting.

In spite of all successes, there is still no direct prove of an event horizon, all observations which hint to it are indirect. ${ }^{7}$ Why is this so important? The existence of an event horizon of the type that a nearby observer can not access to a nearby volume of space, places for some a philosophical problem. If a theory shows an infinity it also may indicate that it reached its limitation. One may ask if there exists the possibility to avoid the existence of such an event horizon, implying to extend the theory of GR.

Another problem is that we already know that GR is incomplete: In its present form it can not be quantized. Meanwhile one tries to find a quantized version, a

This is an Open Access article published by World Scientific Publishing Company. It is distributed under the terms of the Creative Commons Attribution 4.0 (CC-BY) License. Further distribution of this work is permitted, provided the original work is properly cited. 


\section{P. O. Hess}

difficult task, one is allowed to look for a phenomenological treatment of quantum effects. One such effect is related to vacuum fluctuation near large masses (see, for example, ${ }^{8}$ ). These vacuum fluctuations must have an effect on the metric and, for example, a Schwarzschild solution should be modified. Behind these thoughts is a principle which states that masses not only curve space but also change the vacuum properties, which itself modify the metric.

$\mathrm{In}^{9}$ we proposed a possible algebraic extension of GR (leading to the pseudocomplex General Relativity (pc-GR)), which not only requires the appearance of an additional energy-momentum tensor on the right hand side of the Einstein equations, but also included a minimal length. The energy-momentum tensor will be related to vacuum fluctuation, which will be treated phenomenologically.

In section 2 a short review is given on why a pseudo-complex extension makes sense and not others. We also will show that under certain conditions a minimal length appears and in this case the theory reproduces others with a minimal length.

The matter with the minimal length will not be pursued further, principally because no measurable effects can be calculated, it is only of academic value. Instead, in section 3, we will resume some results concerning QPO's and simulations of accretion disks. The clear differences between the predictions in GR compared to pc-GR is shown.

Finally, some new developments concerning the observation of gravitational waves will be presented.

\section{The Pseudo-Complex General Relativity and a Minimal Length}

$\mathrm{In}^{9}$ an algebraic extension of GR was proposed, called pc-GR. The coordinates $x^{\mu}$ $(\mu=0,1,2,3)$ are extended to pseudo-complex coordinates, with $X^{\mu}=x^{\mu}+I y^{\mu}$ and $I^{2}=1$. The last condition is the definition of coordinates. Defining $\sigma_{ \pm}=\frac{1}{2}(1 \pm I)$, the coordinates can also be written as $X^{\mu}=X_{+}^{\mu} \sigma_{+} X_{-}^{\mu} \sigma_{-}$. Because $\sigma_{+} \sigma_{-}=0$, the two spaces separate and can be treated independently. For more details, please consult. $^{10}$

That only a pc-extension makes sense was shown in: ${ }^{11}$ In the weak field limit of GR the pseudo-imaginary part of the propagators are multiplied by $I^{2}=1$, which yields physical propagators. In any other algebraic extension (complex variables, quaternions,..) minimally one $i^{2}=-1$ appears which implies a ghost-propagator, which is not physical.

In the pc-GR the metric is pseudo-complex

$$
g_{\mu \nu}=g_{\mu \nu}^{R}+I g_{\mu \nu}^{I}
$$

where $R$ and $I$ refer to the pseudo-real and pseudo-imaginary component, respectively.

Defining the symmetric $\left(g_{\mu \nu}^{S}=\frac{1}{2}\left(g_{\mu \nu}^{R}-g_{\mu \nu}^{I}\right)\right)$ and antisymmetric $\left(g_{\mu \nu}^{A}=\right.$ $\left.\frac{1}{2}\left(g_{\mu \nu}^{R}-g_{\mu \nu}^{I}\right)\right)$ combination of the pseudo-real and pseudo-imaginary part, the line 
element has the form

$$
\begin{aligned}
d \omega^{2}= & {\left[g_{\mu \nu}^{S}\left(d x^{\mu} d x^{\nu}+d y^{\mu} d y^{\nu}\right)+2 g_{\mu \nu}^{A} d x^{\mu} d y^{\nu}\right] } \\
& I\left[g_{\mu \nu}^{A}\left(d x^{\mu} d x^{\nu}+d y^{\mu} d y^{\nu}\right)+2 g_{\mu \nu}^{S} d x^{\mu} d y^{\nu}\right]
\end{aligned}
$$

i.e., the line element is also pseudo-complex.

Using as the Lagrangian $\mathcal{L}=\sqrt{g}(\mathcal{R}+2 \Lambda)$, with $g$ the determinant of the metric and $\mathcal{R}$ the Riemann scalar (all pseudo-complex), after a standard variational approach $^{10,12}$ and the constraint that $d \omega^{2}$ has to be real, the Einstein equations acquire the form $(c=G=1)$

$$
G_{\mu \nu}=-8 \pi T_{\mu \nu}
$$

with $G_{\mu \nu}$ the Einstein tensor and $T_{\mu \nu}$ an energy-momentum tensor which is required by the theory. On the physical origin of this tensor the theory can not give an answer, because up to now we just extended the GR using pure mathematical arguments. $\mathrm{In}^{8}$ a possible reasoning is given that the origin are due to vacuum fluctuations which each mass provokes in its surroundings, leading to the principle that mass not only curves space but also changes the vacuum properties. Because no quantized theory of GR exists yet, we will recur to a phenomenological approach.

It is possible to show that pc-GR includes a series of theories which contain a minimal length: In flat space one can show that the pseudo-imaginary component of the coordinate is proportional to the 4 -velocity, ${ }^{13}$ i.e. $y^{\mu}=l u^{\mu}$, where $l$ has the dimension of length and has to be added for maintaining units. Assuming also that the metric can be approximately by a real one (then $g_{\mu \nu}^{S}=g_{\mu \nu}$ and $\left.g_{\mu \nu}^{A}=0\right),(2)$ reads

$$
d \omega^{2}=g_{\mu \nu}\left(d x^{\mu} d x^{\nu}+l^{2} d u^{\mu} d u^{\nu}\right) .
$$

This is the line element proposed by Caianiello ${ }^{14}$ and before in a similar way by Born. ${ }^{15}$ Unfortunately, all our efforts to find some observable predictions failed and we concentrated to the case with $l=0$, where the Einstein equations only contain pseudo-real tensors. $\operatorname{In}^{16}$ the pc-Schwarzschild, pc-Kerr and the pc-ReisnerNordström solutions are presented.

\section{QPO's and Simulations of an Accretion Disk}

$\mathrm{In}^{17}$ particles in a circular orbit around a large mass were considered and the redshift as a function of the distance calculated. The main motivation was to describe the QPO's observed in galactic centers with an accretion disk surrounding the central black hole. The accepted interpretation is that a local bright source of light is following the rotation of the accretion disk. From there one can deduce the distance to the central object using GR or pc-GR. However, in order to confirm the distance one has to measure the redshift of the $\mathrm{Fe} \mathrm{K}-\alpha$ line which should give within the theory the same distance. No lines are seen up to now in galactic centers but in several candidates of galactic black holes, a binary system consisting of a black hole with a companion star providing mass to the accretion disk. 


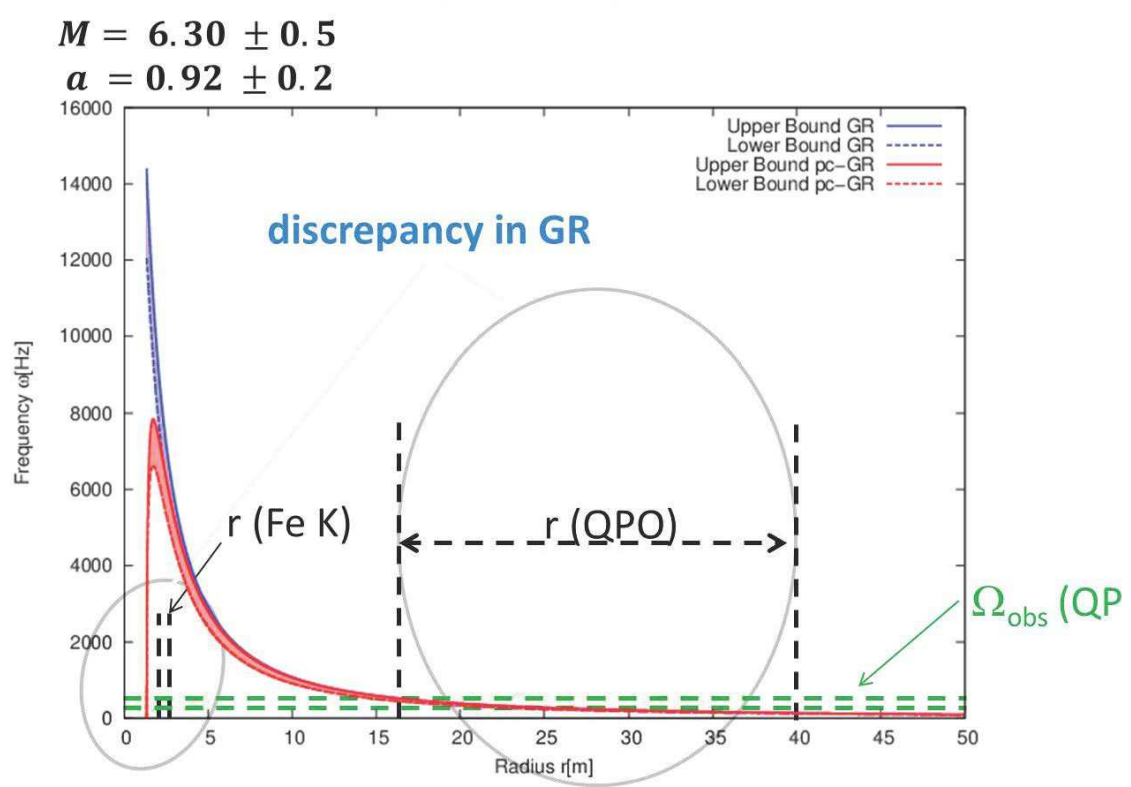

Fig. 1. Orbital frequency, in units of Hertz is plotted as a function in $\mathrm{r}$, for the system GRO J1655-40. The range of $\mathrm{r}$, as deduced through the measurement of the redshift, is indicated by two vertical lines on the left side. The other vertical lines on the right refer to the range of $\mathrm{r}$ deduced in the measurement of the orbital frequency. The dependence of the orbital frequency and the redshift as a function on the relative distance is depicted.

In Fig. 1, one case is shown and the discrepancy between the measured distance and redshift when GR is used, and on Fig. 3 the data are compared to pc-GR. As can be seen, GR does not provide a coincidence but pc-GR does. Of course, this depends on the interpretation of the QPO as being local bright source following the rotation of the accretion disk. If the companion star provokes other types of periodic movements one can probably still reconcile with GR.

Finally, we show the result of a simulation of accretions disks around a massive object. The model proposed by Page and Thorn ${ }^{18}$ is used, which uses the approximations of an infinite thin accretions disk, whose mass can be ignored compared to the mass of the black hole, the emission of energy is only through photons and the transportation of the energy within the accretion disk is through shear forces. In Fig, 3 we compare for the rotational parameter $a=0.9$ the GR result (left panel) with the pc-GR result (right panel). The distinct feature in pc-GR is that a dark ring followed by a bright one appears, absent in GR. The reason for that is the maximum of the orbital frequency which decreases from there on toward the center. At the maximum, the orbital frequency of two neighboring orbitals is very similar. leading to less friction and the dark ring. Further toward the center the change of 


\section{Galact $\mathrm{c}$ binaries in the pc-theory}

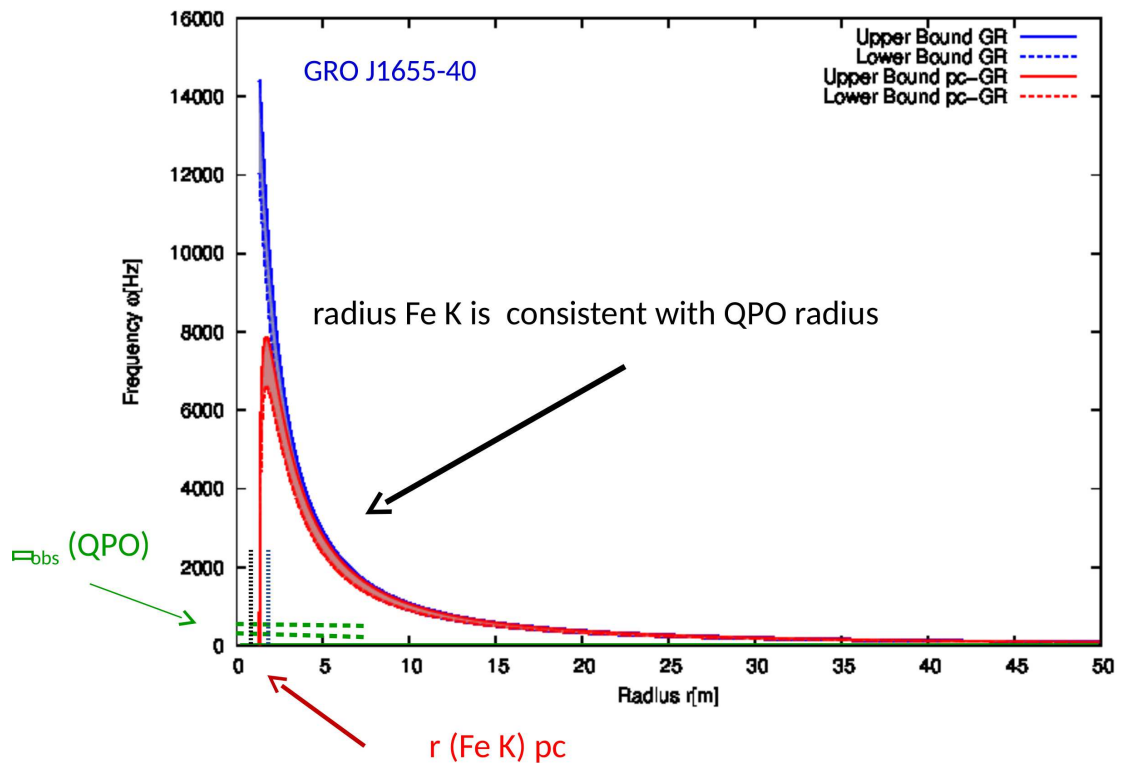

Fig. 2. The same as in Figure 1 but now for pc-GR. (Courtesy from Th. Boller.)

the orbital frequency is strong, leading to the bright ring. This feature only appears for $a>0.4$ while for $a<0.4$ the simulations are similar to GR, with the difference that the overall brightness is larger in pc-GR. Further simulations were presented in. ${ }^{19,20}$

\section{A New Interpretation of the Source of the Observed Gravitational Waves}

Recently the first observation of gravitational waves was reported ${ }^{2-5}$, a wonderful confirmation of Einstein's predictions! $\mathrm{In}^{2}$ the two masses, which finally fused, are approximated by two point masses. The measured frequency and the change of it in time did lead to a chirping mass of about 30 solar masses, corresponding for the case of then GW150914 event to approximately 30 solar masses each. From the amplitude of the gravitational wave the luminosity distance can finally be deduced leading to about $400 \mathrm{Mpc}$.

There is no doubt that finally gravitational waves are observed. However, the

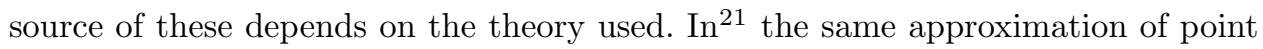
masses was used, with the difference that the gravitational constant depends effectively on the radial distance, due to the accumulation of dark energy toward the center. The relation of the observed frequency $f_{\mathrm{gw}}$ and its change in time leads in pc-GR to 

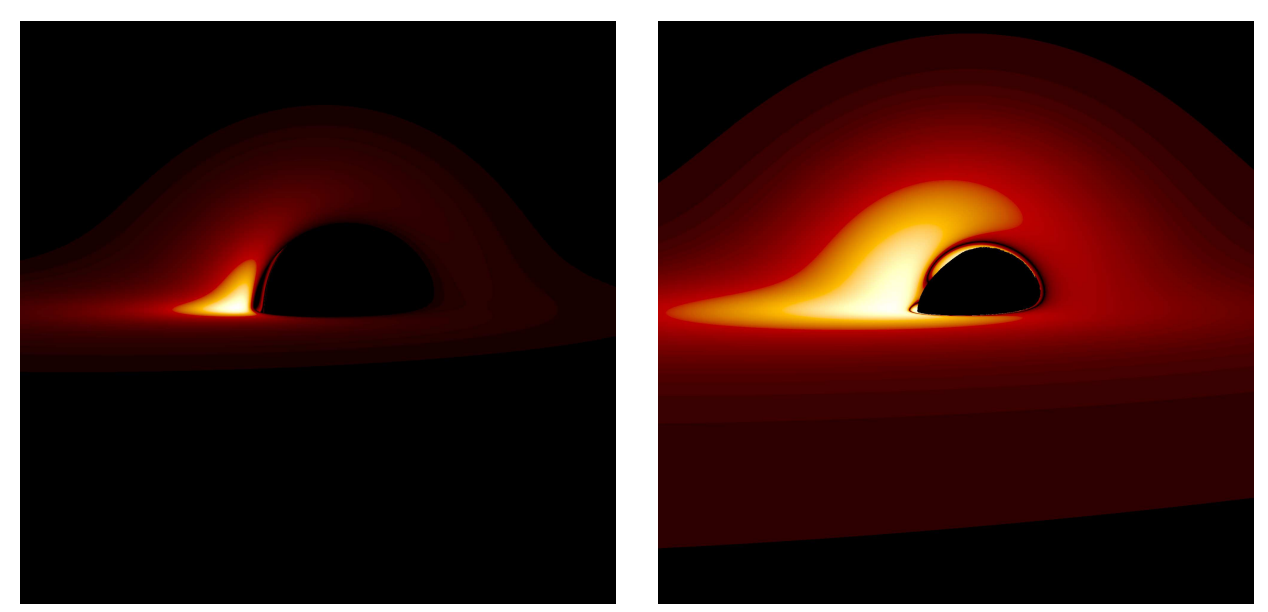

Fig. 3. Infinite, counter clockwise rotating geometrically thin accretion disk around static and rotating compact objects viewed from an inclination of 85 degrees. The left panel shows the original disk model by (Page and Thorne 1974). The right panel shows the results within pc-GR. The spin parameter is $a=0.9 . a$ is given in units of $m$.

$$
\mathcal{M}_{c}=\widetilde{\mathcal{M}}_{c} F_{\omega}(R)=\frac{c^{2}}{G}\left[\frac{5}{96 \pi} \frac{8}{3} \frac{d f_{\mathrm{gw}}}{d t} f_{\mathrm{gw}}^{-\frac{11}{2}}\right]^{\frac{3}{5}}
$$

where $F_{\omega}=1-\frac{3 b}{4}\left(\frac{R_{S}}{2 R}\right)^{2}$ and $R$ is the relative distance of the two stars before merger. The right hand side determines the measured value $\mathcal{M}_{c}=30$. However, this is within pc-GR not the chirping mass but the chirping mass multiplied by a function $F_{\omega}$ which gets small. Due to that the real chirping mass is much bigger, resulting also in larger masses of the participant stars.

As a result, the measured frequency and the change of it with time leads to chirping masses between 4000 and 16000 solar masses and the distance varies between $38000 \mathrm{Mpc}$ and $141000 \mathrm{Mpc}$. This changes the scenario such that the sources are probably centers of primordial galaxies which fused in the formation of larger galaxies.

\section{Conclusions}

In this contribution a brief presentation of the pc-GR was given and the main results were presented:

1) The orbital frequency of a point particle around a large mass shows a maximum approaching the center, falling off after the maximum. This has important consequences for the observed QPO's in galactic black holes, suggesting first observed deviations to GR.

2) The flux of light from the accretion disk is much larger in pcGR than in GR.

3) The pcGR predicts the appearance of a dark ring followed by a bright one in accretion disks with a rapid rotating black hole. 
4) The source of the observed black hole mergers is interpreted within pc-GR as being two central black holes from two primordial galaxies, as the consequence of a former merger of these.

\section{Acknowledgments}

This work belongs to the DGAPA project IN100315. Very useful discussions with Thomas Boller (Max Planck, Garching, Germany) are acknowledged.

\section{References}

1. C. M. Will, Living Rev. Telativ. 9, 3 (2006).

2. B. P. Abbott et al., (LIGO Scientific Collaboration and Virgo Collaboration), Phys. Rev. Lett. 116, 061102 (2016).

3. The LIGO Scientific Collaboration and The Virgo Collaboration, arXiv[grqc]:1602.03840(2016).

4. B. P. Abbott et al., Phys. Rev. Lett. 116, 241103 (2016).

5. B. P. Abbott et al., preprint, June 15, (2016).

6. http://www.eventhorizontelescope.org.

7. M.A. Abramowicz, W. Kluzniak, and J.P. Lasota, Astr. and Astrophys. 396, 31 (2002).

8. M. Visser, Phys. Rev. D 54, 5116 (1996).

9. Peter O. Hess and Walter Greiner, Int. J. Mod. Phys. E 18, 51 (2009).

10. P. O. Hess, M. Schäfer, and W. Greiner, Pseudo-Complex General Relativity, (Springer, Heidelberg, 2015).

11. P. F. Kelly and R. B. Mann, Class. Quant. Grav. 3, 705 (1986).

12. P. O. Hess and W. Greiner, Centennial of General Relativity: A Celebration, Edited by: C. A. Zen Vasconcellos (World Scientific Publishing, Singapore, 2017), p. 97.

13. P. O. Hess, Astron. Nach. 336, 739 (2015).

14. E. R. Caianiello, Nuovo Cim. Lett. 32,65 (1981).

15. M. Born, Proc. Roy. Soc. A 165,291 (1938).

16. G. Caspar, T. Schönenbach, P. O. Hess, M. Schäfer, and W. Greiner, Int. J. Mod. Phys. E 21,1250015 (2012).

17. T. Schönenbach, G. Caspar, P. O. Hess, T. Boller, A. Müller, M. Schäfer, and W. Greiner, MNRAS 430, 2999 (2013).

18. D.N. Page and K. S. Thorne, ApJ, 191, 499 (1974).

19. T. Schönenbach, G. Caspar, P. O. Hess, T. Boller, A. Müller, M. Schäfer, and W. Greiner, MNRAS 442, 121 (2014).

20. P.O. Hess1, M. Algalán, T. Schönenbach, and W. Greiner, Astron. Nachr. 336, 722 (2015).

21. P. O. Hess, MNRAS 462, 2016 (2016). 